\title{
MODELAGEM FUZZY APLICADA NA DETECÇÃO DA VULNERABILDADE À EROSÃO COSTEIRA
}

\author{
Fuzzy modeling applied to coastal erosion vulnerability detection \\ LUCIANA MARIA DA SILVA ${ }^{1}$ \\ RODRIGO MIKOSZ GONÇALVES ${ }^{2}$ \\ MILDE MARIA DA SILVA LIRA ${ }^{3}$ \\ PEDRO DE SOUZA PEREIRA ${ }^{4}$ \\ Universidade Federal de Pernambuco - UFPE \\ Centro de Tecnologia e Geociências - CTG \\ 1,2 Departamento de Engenharia Cartográfica, Programa de Pós-Graduação em \\ Ciências Geodésicas e Tecnologias da Geoinformação \\ ${ }^{3}$ Departamento de Engenharia Elétrica \\ ${ }^{4}$ Departamento de Oceanografia \\ lumasilva15@gmail.com; rodrigo.mikosz@ufpe.br; milde@ufpe.br; \\ psppraias@gmail.com
}

\begin{abstract}
RESUMO
O crescimento da urbanização vem provocando grandes transformações nas relações sociais e morfológica das áreas costeiras. $\mathrm{O}$ presente trabalho tem como objetivo modelar massa de dados de diversas fontes como GNSS (Global Navigation Satellite Systems) e sensoriamento remoto para posteriormente prover a espacialização da vulnerabilidade costeira à erosão utilizando a lógica fuzzy. O método aplicado para análise da vulnerabilidade utilizou variáveis linguísticas, partições fuzzy, intervalos e análises paramétricas que caracterizam a modelagem fuzzy. Após análise, os níveis de vulnerabilidade à erosão costeira ao longo da linha de costa nas cidades do Recife e Jaboatão dos Guararapes no litoral de Pernambuco apresentaram os seguintes resultados: $33,33 \%$ da linha de costa possuem vulnerabilidade baixa, $38,15 \%$ possui vulnerabilidade moderada, $14,26 \%$ vulnerabilidade alta e $14,26 \%$ muito alta.

Palavras-chave: Vulnerabilidade à Erosão Costeira; Cartografia Costeira; Modelagem Fuzzy; Linha de Costa.
\end{abstract}




\section{ABSTRACT}

The advance of urbanization has caused substantial transformations in the social and morphological aspects of coastal areas. The present study aims at modeling data from several sources, such as GNSS (Global Navigation Satellite Systems), and remote sensing and subsequently to provide the spacialization of coastal vulnerability to erosion, by using fuzzy logic. In the applied method for vulnerability analysis, linguistic variables, fuzzy partitions, intervals and parametric analysis that characterize fuzzy modeling were used. After analyzing the levels of vulnerability to coastal erosion along the shoreline in the cities of Recife and Jaboatão dos Guararapes at the coast of Pernambuco, the following results were achieved: $33.33 \%$ of the coastline has low vulnerability while, $38.15 \%$ moderate vulnerability, $14.26 \%$ high vulnerability and, $14.26 \%$ very high vulnerability.

Keywords: Vulnerability to Coastal Erosion; Coastal Mapping; Fuzzy Modeling; Shoreline.

\section{INTRODUÇÃO}

A linha de costa é considerada como uma das feições geomorfológicas mais dinâmicas do nosso planeta (BOAK e TURNER, 2005; ALMEIDA, 2008; ROCHA et al., 2008). Seu monitoramento é necessário para dar suporte ao gerenciamento costeiro. Em um ambiente costeiro verifica-se em vários locais o processo de erosão costeira, resultado da combinação de fatores naturais como: tempestades, correntes marítimas, mudanças sazonais além da ação humana (explorações costeiras e as atividades de engenharia) (ROCHA et al., 2008; HARLEY et al., 2011).

O mapeamento costeiro vem se beneficiando do estado da arte do GNSS onde um dos grandes desafios é aplicar um método de posicionamento para obtenção precisa de feições costeiras relacionadas a um determinado instante de tempo. Rocha et al. (2008) retratam a aplicação do posicionamento GPS (Global Positioning System) tridimensional para localizar a linha de costa e proporcionam a obtenção do modelo de elevação digital do terreno para estudos costeiros. Tanajura et al. (2011) comparam a acurácia do posicionamento GNSS para zonas costeiras testando diferentes métodos de posicionamento e processamento de dados entre eles: o método absoluto cinemático, relativo cinemático e diferencial (RTK e DGPS) . Em muitos estudos costeiros a falta de informação cartográfica temporal pode gerar dificuldade no diagnóstico de uma determinada área, sendo muito comum em modelagens costeiras a combinação de informações de diferentes fontes. Neste sentido, Gonçalves et al. (2010) apresentam as redes neurais como alternativa para modelos preditivos do comportamento posicional da linha de costa. Em Gonçalves et al. (2012) é possível verificar uma comparação entre modelos de tendência, como por exemplo, a estimativa robusta e o modelo de regressão linear, assim como o seu comportamento tendencial aplicado ao mesmo conjunto de dados temporais da linha de costa. 
A partir da década de 60, as praias de Boa Viagem e Pina localizadas no município de Recife-PE e as praias de Barra de Jangada, Candeias e Piedade localizadas no município de Jaboatão dos Guararapes-PE vêm sendo urbanizadas próximo à linha de costa. Diante das construções cada vez mais sobre a pós-praia e até mesmo do estirâncio, observam-se problemas de vulnerabilidade à erosão costeira.

Esses processos dão origem às praias urbanas que, segundo Smith (1991), são aquelas que circundam cidades, bairros ou balneários consolidados de diversos níveis de desenvolvimento. Ou seja, em alguns casos são caracterizadas como praias sob ocupação desordenada que interferem em processos naturais podendo causar erosão.

Este trabalho utiliza os princípios de uma modelagem fuzzy para elaboração de mapas temáticos da vulnerabilidade costeira baseada em variáveis disponíveis para os estudos abrangendo um trecho da praia do Pina e as praias da Boa Viagem e Piedade.

\section{VULNERABILIDADE À EROSÃO COSTEIRA}

É comum pessoas não especialistas em áreas costeiras utilizarem como sinônimos riscos e vulnerabilidades para avaliar o potencial de risco dos eventos naturais ao longo da costa. Boruff et al. (2005); Marandola Jr. e Hogan (2009) descrevem a definição desses conceitos, onde risco é a probabilidade de um evento ocorrer, enquanto vulnerabilidade é definido como os fatores que aumentam ou atenuam os efeitos de um extremo natural, evento tecnológico ou humano e os fatores que diminuem a comunidade ou a capacidade do indivíduo para se recuperar após o evento ocorrer. Esse conceitos também são destacados em Mazzer et al. (2008).

O problema da erosão costeira é um processo natural que pode ter causas antrópicas, podendo se tornar um problema ocasionando a perda de patrimônios. Morais et al. (2008) relatam que nos estudos da erosão costeira em Maceió, os imóveis localizados nos primeiros $50 \mathrm{~m}$ desvalorizaram-se economicamente. Os usuários desta praia atribuíram a dificuldade ao acesso e impossibilidades na prática do banho.

A erosão costeira ocorre em vários lugares do mundo podendo alcançar estágios críticos, por exemplo, na Espanha, em trechos do litoral, a urbanização não deixou espaço suficiente para a dinâmica costeira ocasionando prejuízos ambientais, comerciais e recreativos (DOODY, 2001). No estado de Delaware localizado na costa leste dos Estados Unidos, o enorme valor das propriedades ao longo da costa e o valor econômico da indústria do turismo costeiro combinaram para criar um problema de gestão de recursos naturais que é particularmente difícil de resolver, pois na medida em que a indústria do turismo nessa área se expande, há o aumento de barreiras para evitar o avanço do mar devido à erosão costeira (DANIEL, 2001).

Nos contextos apresentados anteriormente, observa-se que a vulnerabilidade à erosão costeira está associada a diversos processos morfodinâmicos assim como

Bol. Ciênc. Geod., sec. Artigos, Curitiba, v. 19, no 4, p.746-764, out-dez, 2013. 
antrópicos, onde se pode utilizar uma ampla gama de métodos para analisar o grau de vulnerabilidade. Em Bryan et al. (2001), a vulnerabilidade costeira está relacionada ao nível médio do mar por uma complexa interação entre quatro parâmetros fisicos ambientais (elevação, índices de erosão, aspectos físicos e inclinação). Para Clark et al. (1998), a vulnerabilidade significa a inclusão de tipos múltiplos em perigo e a análise de riscos em um determinado local, equanto para Wu et al. (2010), a vulnerabilidade é expresssa em termos do potencial de exposição a um perigo físico.

Atualmente através de um determinado conjunto utilizando dados qualitativos e quantitativos vêm se desenvolvendo métodos para análise da vulnerabilidade costeira à erosão. Para as variáveis são atribuídos pesos proporcionais à influência da vulnerabilidade em um modelo analítico objetivando gerar índices numéricos que resultam em mapas temáticos temporais da vulnerabilidade ao longo de uma área costeira, como pode ser visto em Mazzer et al. (2008); Mallmann e Araújo (2010); Raposeiro e Ferreira (2010). Sendo assim, diversos estudos vêm buscando formas de combater a erosão costeira, por exemplo, Ryabchuk et al. (2010) apresentam um estudo de longo e curto prazo da erosão costeira ao longo da linha de costa no leste do Golfo da Finlândia. Hapke e Plant (2010) apresentam um modelo probabilístico Bayesiano para tentar prever a erosão costeira do penhasco ao norte de São Diego, Califórnia onde se verificam o aumento gradual do nível do mar e eventos episódicos ocasionados por tempestades, entre outras pesquisas.

\section{LÓGICA FUZZY}

A lógica fuzzy permite soluções aproximadas. Lotfi Asker Zadeh foi o pioneiro na criação da lógica fuzzy que combinou os conceitos da lógica clássica e os conjuntos de Lukasiewicz (graus de pertinência sendo: 0, $1 / 2 \mathrm{e} 1$, mais tarde expandiu um número infinito de valores entre 0 e 1 ). Os conceitos fundamentais de lógica fuzzy podem ser visto em Zadeh (1965).

O raciocínio fuzzy é formado por três etapas: a fuzzificação, a inferência e a defuzzificação.

- Fuzzificação: transforma as variáveis do problema em valores fuzzy, ou seja, valores numéricos em termos da linguagem natural.

- Inferência é a etapa mais importante do raciocínio fuzzy, pois é mediante ela que é feita a tomada da decisão. Também é efetuado o raciocínio permitindo conclusões a partir de fatos conhecidos (min-max) e das variáveis linguísticas de entrada e saída.

- Defuzzificação: transforma resultado fuzzy em resultado escalar, ou seja, transforma o dado nebuloso em dado quantitativo (consiste em retornar valor numérico na faixa estipulada pela lógica fuzzy). Os métodos mais utilizados para defuzzificação ou conversão fuzzy em escalar são: Centro de Massa (CoM), Média do Máximo (MoM), Centro da Área (CoA), Média Ponderada (MP). Mais detalhes em Gomide e Gudwin (1994); Fernandes 
(2005); Rezende (2005); Shaw e Simões (2007); Silva (2008); Silva (2013).

Várias pesquisas em Cartografia e Geodésia têm utilizado a lógica fuzzy como exemplo, Antunes et al. (2003) explicitam a classificação de imagens digitais de alta resolução com base na análise orientada a objetos, considerando descritores espectrais, forma, textura e posição usando as regras fuzzy em uma área teste na zona agrícola no município de Nova Esperança-PR, onde foi classificada a mata ciliar ao longo do Rio Porecatú; Lazzarotto et al. (2005) utilizaram os indicadores e sistema de inferência fuzzy como avaliação da qualidade de base cartográfica; Kersting e Centeno (2007) fazem a categorização dos objetos por meio de classificador fuzzy, a partir da definição das funções de pertinência baseadas nos descritores de forma, textura e relação entre os objetos; Ximenes et al. (2008), verificam a eficácia do modelo da dinâmica do desmatamento na Amazônia mediante a simulação do índice de similaridade fuzzy elevado; Fernandes et al. (2010) utilizam a lógica fuzzy e os sistemas de informações geográficas como apoio a decisão do planejamentos do uso do espaço, onde elaboraram um mapa de sugestão, utilizando o conjunto fuzzy para analisar o resultado dos mapas entre outras pesquisas.

\section{4. ÁREA DE ESTUDO}

A cidade do Recife, localizada nas coordenadas ( $\left.08^{\circ} 03^{\prime} 14^{\prime \prime} \mathrm{S}, 34^{\circ} 52^{\prime} 51^{\prime \prime} \mathrm{W}\right)$, conforme dados do censo de 2010, conta com uma população de 1.537 .704 habitantes e apresenta uma superfície territorial de 218,498 $\mathrm{km}^{2}$,com 7.037,6 hab $/ \mathrm{km}^{2}$. A cidade de Jaboatão dos Guararapes limita-se ao norte do município do Recife, localizada nas coordenadas $\left(08^{\circ} 06^{\prime} 43^{\prime \prime} \mathrm{S}, 35^{\circ} 00^{\prime} 55^{\prime} \mathrm{W}\right)$, conforme dados do censo de 2010, conta com uma população de 644.620 habitantes, apresenta uma superfície territorial de $258,566 \mathrm{~km}^{2}$, com 2.493,06 hab $/ \mathrm{km}^{2}$.

Segundo Aureliano (2000) a caracterização da ocupação do município ocorreu por meio de loteamentos residenciais, industriais e comerciais, sendo que, nas últimas décadas, houve grande ascensão na construção de edifícios, condomínios e hotéis ao longo da orla.

A Tabela 1 mostra os dados de área, população e densidade demográfica em 2010 dos municípios na malha urbana de Recife e Jaboatão dos Guararapes, totalizando aproximadamente três milhões e meio de habitantes. Segundo Manso et al. (2006), o litoral pernambucano foi delimitado e setorizado com base na sua situação geográfica e política em 21 municípios, distribuídos numa faixa de $187 \mathrm{~km}$ que se estende desde o município de Goiana, ao norte, até o município de São José da Coroa Grande, ao sul. A costa pernambucana apresenta baixa altitude, chegando a atingir, em vários pontos, altitudes inferiores ao nível médio do mar.

A Figura 1 apresenta o mapa de localização do Estado de Pernambuco na Região Nordeste do Brasil, a ampliação do Estado e em particular um polígono que define os municípios de Recife e Jaboatão dos Guararapes. 
Tabela 1 - Área total e população dos municípios na malha urbana.

\begin{tabular}{c|c|c|c}
\hline Municípios & $\begin{array}{c}\text { População } \\
\text { (hab) }\end{array}$ & $\begin{array}{c}\text { Área } \\
\text { (km2) }\end{array}$ & $\begin{array}{c}\text { Densidade Demográfica } \\
\text { (hab/km2) }\end{array}$ \\
\hline Cabo de Santo Agostinho & 185.025 & 446,578 & 414,32 \\
\hline Camaragibe & 144.466 & 51,194 & $2.821,93$ \\
\hline Jaboatão dos Guararapes & 644.620 & 258,566 & $2.493,06$ \\
\hline Moreno & 56.696 & 196,071 & 289,16 \\
\hline Olinda & 377.779 & 41,659 & $9.068,36$ \\
\hline Paulista & 300.466 & 97,364 & $3.086,01$ \\
\hline Recife & 1.537 .704 & 218,498 & $7.037,61$ \\
\hline São Lourenço da Mata & 102.895 & 262,157 & 392,49 \\
\hline Total & 3.349 .651 & 1572,087 & $2.130,70$ \\
\hline
\end{tabular}

Fonte: IBGE (2010)

Figura 1 - Mapa de Localização da Área de Estudo: Recife e Jaboatão dos Guararapes.

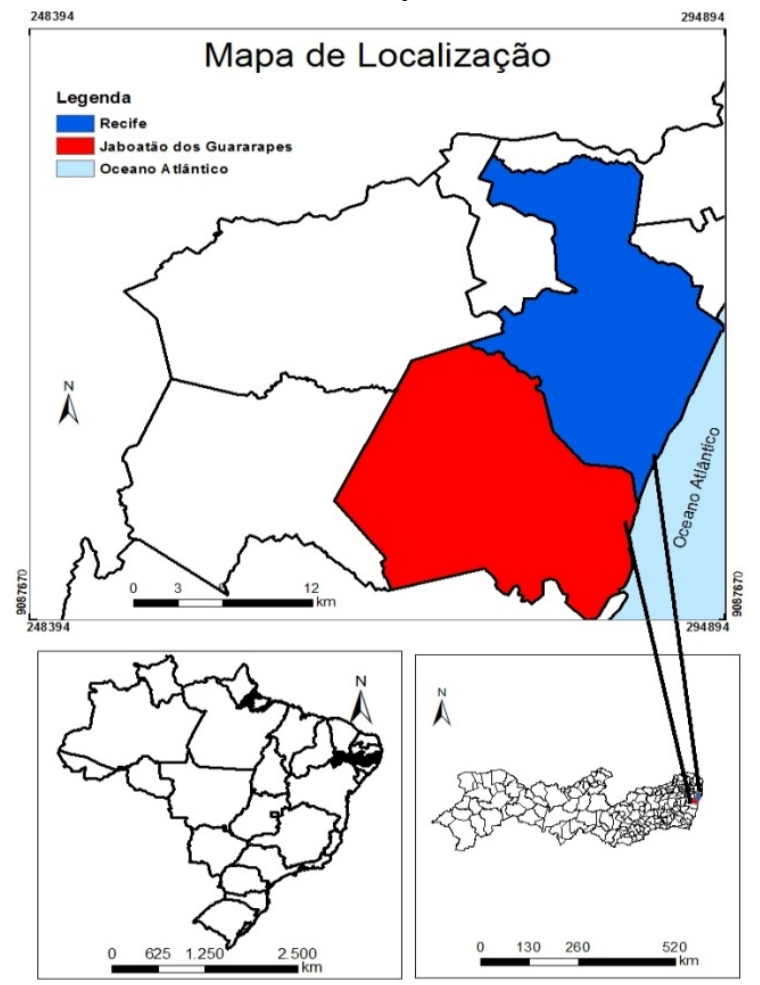

Bol. Ciênc. Geod., sec. Artigos, Curitiba, v. 19, nº 4, p.746-764, out-dez, 2013. 
O litoral do setor do núcleo metropolitano de Pernambuco é formado pelos municípios de Jaboatão dos Guararapes, Olinda e Recife totalizando aproximadamente uma faixa costeira de $30 \mathrm{~km}$. Limitando-se ao norte com o município de Paulista e ao sul com o município de Cabo de Santo Agostinho.

As praias da região metropolitana do Recife em questão estão sujeitas a ondas predominantemente oriundas do quadrante leste e sudeste com altura média em torno de 1 a $1,5 \mathrm{~m}$ e período de 5 a $7 \mathrm{~s}$. As marés nessa porção do litoral brasileiro são classificadas como meso-maré com altura máxima em torno de $2,8 \mathrm{~m}$. Tal variação na altura da maré somada a presença de recifes na plataforma interna modelam a altura das ondas incidentes sobre a costa, modificando o perfil praial que apresenta um terraço de maré baixa bem desenvolvido, com predomínio de sedimentos quartzosos de granulometria média e presença material biogêncio, mais detalhes em Manso et al. (2006).

A cidade de Recife tem seu litoral formado pelas praias do Pina e Boa Viagem que totalizam aproximadamente $8,5 \mathrm{~km}$ de extensão, enquanto Jaboatão é formado pelas praias de Barra de Jangada, Candeias e Piedade que totalizam aproximadamente $8 \mathrm{~km}$.

\section{METODOLOGIA}

Com a organização e resgate de informações temporais obtidas através de imagens orbitais dos satélites artificiais: Quickbird (2005) e Geoeye (2011) e levantamentos geodésicos cinemáticos relativos da linha de costa utilizando GNSS em 2007, 2009, 2010 e 2012 foi possível criar um banco de dados geográfico para extrair informações do processo de vulnerabilidade da área costeira. A seguir são apresentados os materiais e detalhes dos métodos.

\subsection{Materiais}

Este estudo foi desenvolvido utilizando como materiais os seguintes recursos:

- Imagem orbital do satélite artificial: Quickbird (2005) com as seguintes características: resolução espectral por bandas (em $\mu \mathrm{m})$ : B1: $0,45-0,52 \mu$ - azul; B2: 0,52 - 0,60 $\mu$ - verde; B3: 0,63-0,69 $\mu$-vermelho; B4: 0,76$0,90 \mu \mathrm{m}$ - infravermelho; B5: 0,45 - 0,90 $\mu \mathrm{m}$ pancromática e resolução espacial para o modo pancromático de $60 \mathrm{~cm}$ e 2,4m para as bandas B1, B2 e B3.

- Imagem orbital do satélite artificial: Geoeye (2011) com as seguintes características: resolução espectral por bandas (em $\mu \mathrm{m})$ : PAN: $450-800$ $\mu \mathrm{m}$-pancromática; MS: 450 - $510 \mu \mathrm{m}$ - multiespectral; MS: $510-580 \mu \mathrm{m}$ - multiespectral; MS: 655 - $690 \mu \mathrm{m}$ - multiespectral; MS: 780 - $920 \mu \mathrm{m}-$ multiespectral e resolução espacial de $0,41 \mathrm{~m}$ no modo pancromático e $1,65 \mathrm{~m}$ nos demais. A correção geométrica das imagens de satélite utilizadas foi compatível com a escala 1:10.000. 
- Levantamentos geodésicos da linha de costa utilizando GNSS em 2007, 2009, 2010 e 2012. O nível de precisão do método de levantamento cinemático relativo com pós-processamento é em torno de $10 \mathrm{~cm}$ podendo alcançar poucos centímetros caso as condições sejam favoráveis (cobertura de satélites, ausência de multicaminho, distância da linha de base no processamento, etc) (SEEBER, 2003).

- Aquisição de dados cartográficos para modelagem fuzzy da vulnerabilidade costeira através do processo de fotointerpretação e vetorização das imagens de satélite artificiais.

\subsection{Métodos}

Foi realizado a divisão setorial da área de estudo para análise da Modelagem Fuzzy a partir da interpretação visual das imagens e visita em campo, onde as praias de Boa Viagem e Piedade além de um trecho da praia do Pina foram dividida em 6 setores. $\mathrm{O}$ critério para divisão setorial foram as semelhanças nas características geomorfológicas da pós-praia considerando o ambiente praial que são apresentadas e descritas para cada setor. Destaca-se que um desses setores se diferencia dos demais pela construção de enroncamento sobre a linha de costa.

Como pode ser observado na Figura 2, os setores são distribuídos e localizados de Norte para Sul. O Setor 1 inicia-se na praia do Pina e a parte norte da praia de Boa Viagem, onde o seu inicio está localizado no fím do enrocamento de Brasília Teimosa e segue em direção sul com uma extensão de 3060 m. É caracterizado por uma pós-praia bem desenvolvida, com a presença de dunas vegetadas e com uma distância entre a linha de costa e o primeiro obstáculo urbano entre 120 e 40 metros, considerada como estável e moderada.

Seguindo ao Setor 2 localizado no centro da praia da Boa Viagem, iniciando no final das dunas frontais depois do Terceiro Jardim da Avenida Boa Viagem e segue para o sul com uma extensão de $1485 \mathrm{~m}$ até o cruzamento da Av. Boa Viagem com a rua Bruno Veloso. É caracterizado por uma pós-praia sem a presença de dunas e sem vegetação rasteira, mas sem ocorrência de erosão costeira, e com uma distância média entre a linha de costa e o primeiro obstáculo urbano de aproximadamente 40 metros.

O Setor 3 ao norte do início do enrocamento da praia da Boa Viagem iniciando no cruzamento da Av. Boa Viagem com a rua Bruno Veloso e segue para o sul por 410 metros até o início do enrocamento de Boa Viagem. Este Setor caracteriza-se por uma pós-praia próxima ao enrocamento reduzida a menos de 10 metros, com indicações claras de erosão costeira e tem uma região de pós-praia aplainada.

A área com enrocamento de rochas, na Figura 2, está destacada como setor 4, tendo uma extensão de $2400 \mathrm{~m}$. Esta área caracteriza-se com indicações claras de erosão costeira. Seguindo ao Setor 5 ao sul da construção do enrocamento da praia da Boa Viagem inicia-se no final do enrocamento e segue para o sul por uma extensão de $2.882 \mathrm{~m}$. Sendo desta extensão total $794 \mathrm{~m}$ pertencentes a praia da Boa 
Viagem e $2088 \mathrm{~m}$ localizados na praia de Piedade. Este setor apresenta uma face praial bem desenvolvida caracterizada por uma praia arenosa em média $20 \mathrm{~m}$, sem indício de erosão costeira.

Figura 2 - Mapa de Setores ao longo da Faixa Litorânea da praia da Boa Viagem e de Piedade.

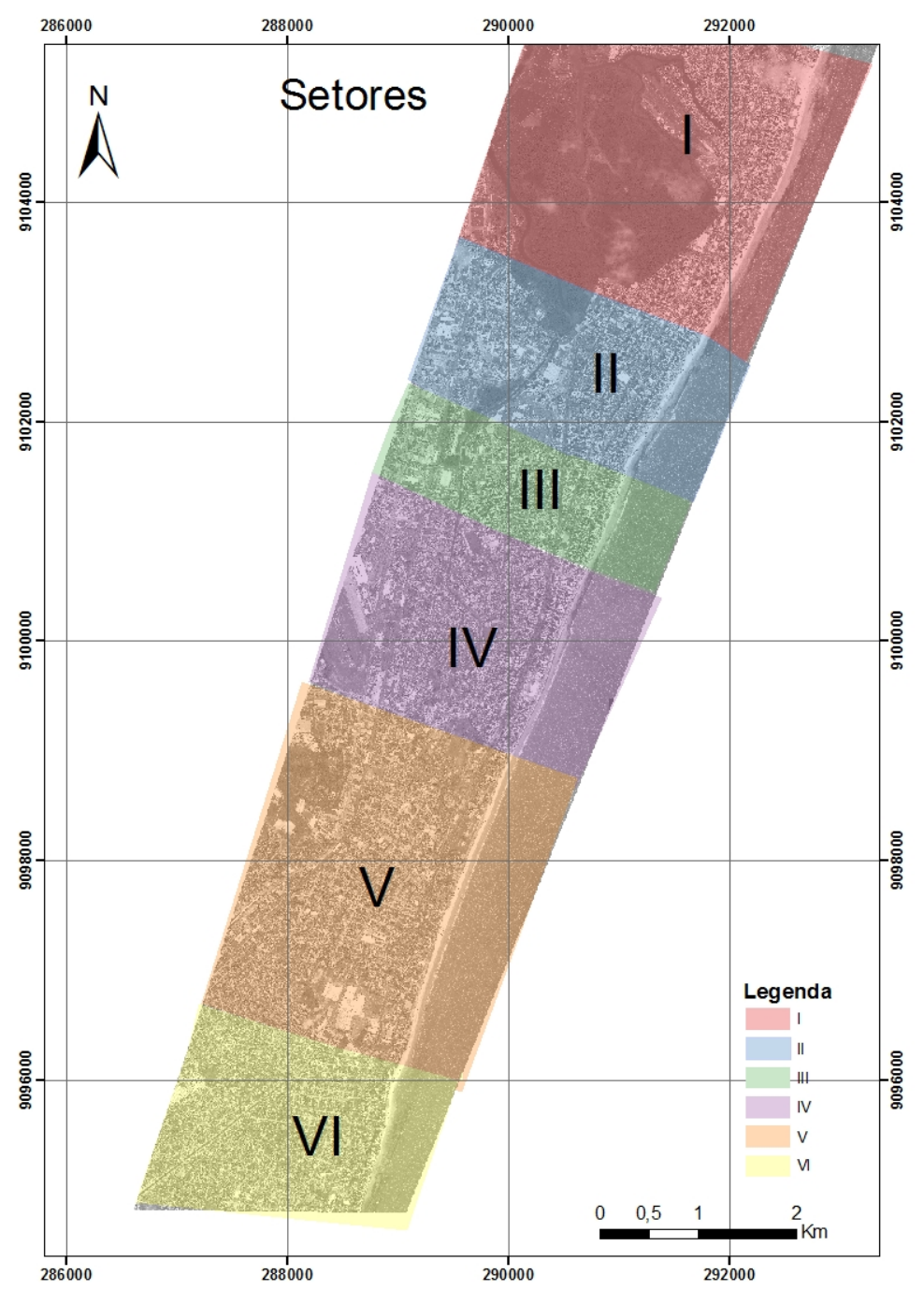

Bol. Ciênc. Geod., sec. Artigos, Curitiba, v. 19, no 4, p.746-764, out-dez, 2013. 
O Setor 6 segue em direção sul da praia de Piedade e possui uma extensão de $2.022 \mathrm{~m}$ até o início do enrocamento da praia de Candeias. Este setor apresenta uma face praial bem desenvolvida, porém na direção sul este setor apresenta uma linha de costa menos retilínea que os setores anteriores. E no final do setor há um estreitamento da face praial.

Ainda poder-se-ia acrescentar outro setor na praia de Piedade ao norte da praia de Candeias, mas optou-se não mapear a linha de costa, pois ela encontra-se em contato direto com as construções e assim os problemas de erosão costeira estão presentes exigindo constantes obras de recuperação e manutenção.

A organização dos dados da área de estudo foi o suporte para a definição da metodologia adotada. $\mathrm{O}$ processo de análise de vulnerabilidade à erosão costeira foi desenvolvido utilizando uma abordagem semi-quantitativa, na qual todos os atributos qualitativos e quantitativos foram codificados em variáveis linguísticas (1, 2, 3 e 4), possibilitando a análise do grau de influência de cada partição fuzzy.

O mecanismo de inferência utilizado foi o de Mamdani, devido sua facilidade na formação das regras lógicas além de permitir o uso de muitas variáveis de saídas. Os dados numéricos utilizados como entradas foram obtidos pelo processo de fotointerpretação e vetorização. Como equação de pertinência foi utilizada a inferência Máx-Min, onde o Máx é formado da união e o Min é formado da intersecção na formação das regras. Para realização da defuzzificação foi utilizado o Centro da Área. Outras opções de inferência e defuzzificação foram analisadas porém os resultados não foram satisfatórios.

O Modelo Fuzzy (MF) necessita de alguns elementos tais como: variáveis de entrada, regras e variáveis de saídas. Para criação do MF foram inseridas seis variáveis de entrada que estão associadas a fatores condicionantes a vulnerabilidade; foram estabelecidas algumas simulações e por meio destas trabalhou-se com a modelagem com 114 regras que fazem parte do processo de inferência fuzzy; e foi estabelecida uma variável de saída que é a Classificação Temática da Vulnerabilidade à Erosão.

As variáveis identificadas nos mapas de setores formam a base de dados de entrada para o modelo fuzzy. Observando os valores numéricos das variáveis e suas funções definiram-se os termos primários de partições fuzzy. Além dos mapas de setores foram construídos mapas. que identificaram os termos primários das partições fuzzy.

A Tabela 2 apresenta os termos primários das partições fuzzy que foram definidos para cada variável. Foram adotadas seis variáveis agrupadas em quatro categorias. Ressalta-se que na escolha das variáveis de entrada e dos termos primários consideraram-se as informações coletadas da área costeira e os mapas construídos. 
Tabela 2 - Variáveis linguísticas utilizadas como dados de entrada e seus termos primários das partições fuzzy correspondentes utilizadas na Lógica Fuzzy.

\begin{tabular}{|c|c|c|c|c|}
\hline Lista para Índices de & \multicolumn{4}{|c|}{ Termos Primários das partições Fuzzy } \\
\hline Descritores & 1 & 2 & 3 & 4 \\
\hline \multicolumn{5}{|l|}{ Condições Morfológicas } \\
\hline $\begin{array}{l}\text { Pós Praia } \\
\text { (Área) }\end{array}$ & $\begin{array}{c}\text { Ampla } \\
(>60 \mathrm{~m} 2)\end{array}$ & $\begin{array}{l}\text { Moderada } \\
(30-60 \mathrm{~m} 2)\end{array}$ & $\begin{array}{c}\text { Estreita } \\
(0- \\
30 \mathrm{~m} 2)\end{array}$ & $\begin{array}{l}\text { Ausente } \\
(0 \mathrm{~m} 2)\end{array}$ \\
\hline \multicolumn{5}{|l|}{ Atributos Naturais } \\
\hline Vegetação & $\begin{array}{l}\text { Rasteira } \\
(0-0,5 \mathrm{~m})\end{array}$ & $\begin{array}{c}\text { Arbustiva } \\
(0,5 \mathrm{~m}-2 \mathrm{~m})\end{array}$ & $\begin{array}{l}\text { Arbórea } \\
(>2 \mathrm{~m})\end{array}$ & $\begin{array}{l}\text { Ausente } \\
(0 \mathrm{~m})\end{array}$ \\
\hline \multicolumn{5}{|l|}{ Processos Costeiros } \\
\hline $\begin{array}{l}\text { Linha de Costa* } \\
\text { (Distância) }\end{array}$ & $\begin{array}{l}\text { Estável } \\
(>60 \mathrm{~m})\end{array}$ & $\begin{array}{l}\text { Moderada } \\
(30-60 \mathrm{~m})\end{array}$ & $\begin{array}{l}\text { Instável } \\
(0-30 \mathrm{~m})\end{array}$ & $\begin{array}{l}\text { Ausente } \\
(0 \mathrm{~m})\end{array}$ \\
\hline \multicolumn{5}{|l|}{ Processos Antrópicos } \\
\hline $\begin{array}{c}\text { Enrocamento** } \\
\text { (Presença em Km) }\end{array}$ & $\begin{array}{l}\text { Ausente } \\
0 \mathrm{~km} \\
\end{array}$ & $\begin{array}{c}\text { Moderado } \\
(0,5-1 \mathrm{~km}) \\
\end{array}$ & $\begin{array}{c}\text { Alto } \\
>1 \mathrm{~km} \\
\end{array}$ & - \\
\hline Benfeitorias de Lazer** & $\begin{array}{l}\text { Ausente } \\
>50\end{array}$ & $\begin{array}{l}\text { Adjacente } \\
(30-50)\end{array}$ & $\begin{array}{l}\text { Presente } \\
(0-30)\end{array}$ & - \\
\hline Urbanização & $\begin{array}{l}\text { Distante } \\
>70\end{array}$ & $\begin{array}{l}\text { Moderada } \\
40-70\end{array}$ & $\begin{array}{l}\text { Próxima } \\
0-40\end{array}$ & $\begin{array}{c}\text { Sem } \\
\text { Ocupação } \\
0\end{array}$ \\
\hline \multicolumn{5}{|c|}{ Saída } \\
\hline $\begin{array}{c}\text { Classificação da } \\
\text { Vulnerabilidade à erosão }\end{array}$ & Baixo & Moderado & Alto & $\begin{array}{l}\text { Muito } \\
\text { Alto }\end{array}$ \\
\hline
\end{tabular}

* Baseado na Lei de Gerenciamento Costeiro de Pernambuco, considerado a distância mínima.

** Cálculos realizados pelo do processo de fotointerpretação

\section{RESULTADOS}

A partir das análises das variáveis de entrada onde se realizou a verificação da coerência do modelo fuzzy e a influência das variáveis na análise da vulnerabilidade, criou-se células (quadriculadas) de $0,25 \mathrm{~km} 2$ nos setores onde foi realizado a análise da vulnerabilidade através da modelagem fuzzy.

A Figura 3 mostra as variáveis de entrada e os seus termos primários de partição fuzzy que foram utilizados para analisar a modelagem fuzzy.

O Quadro 1 foi criado mediante a análise da Figura 3 identificando as variáveis pelo processo de fotointerpretação e quantificadas por meio de vetorização, para serem utilizadas como entrada da modelagem fuzzy. Os dados do 
Quadro 1 são as variáveis e os termos primários de partição fuzzy identificadas em cada linha de célula ao longo das praias de Boa Viagem e Piedade.

Figura 3 - Variáveis de entrada e os termos primário das partições fuzzy codificadas em cores para análise do desempenho da Modelagem Fuzzy.

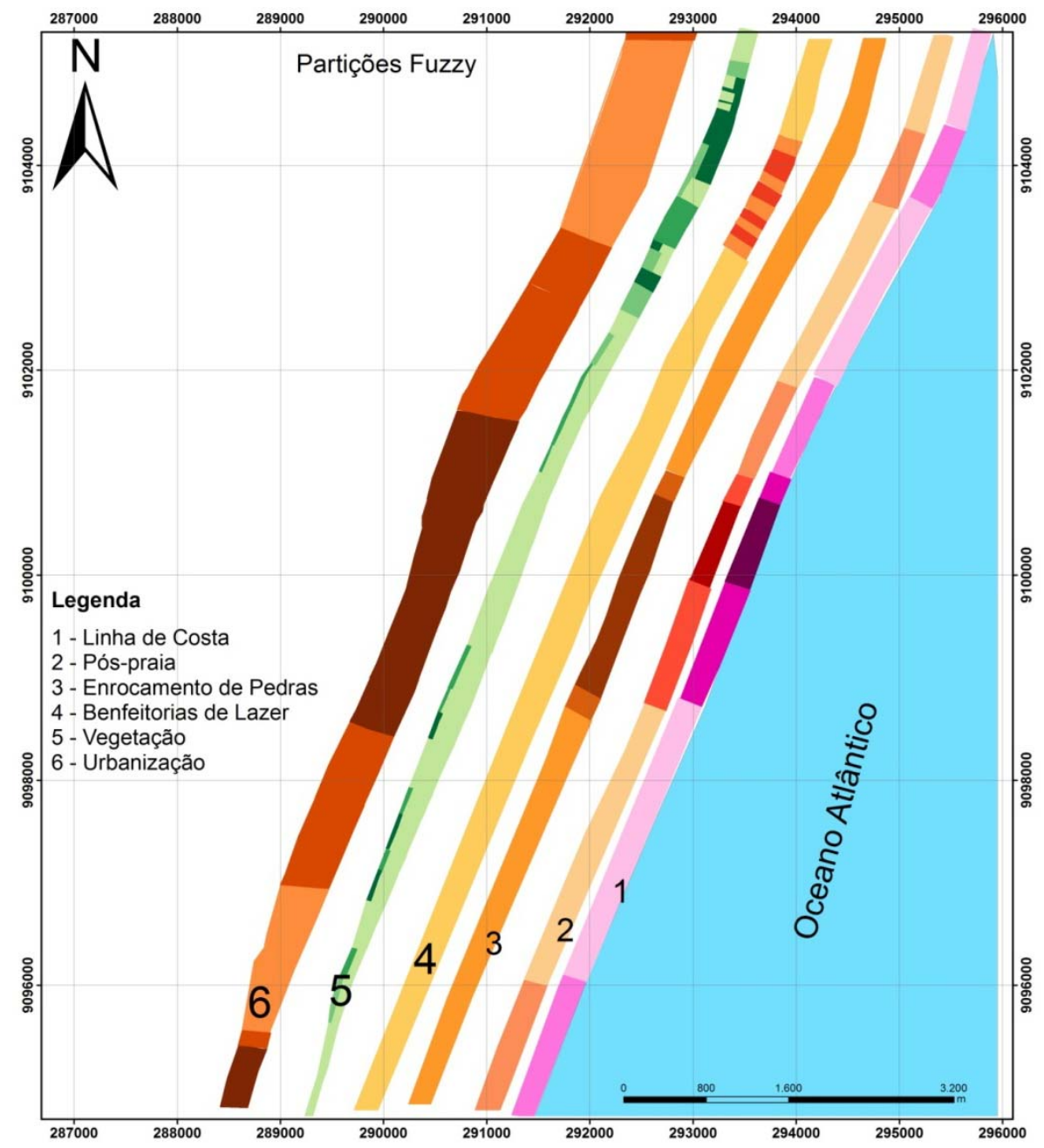

Por meio dos termos primários da partição fuzzy identificados na Figura 3 e construídos no Quadro 1 criaram-se os limites que foram inseridos na interface da Toolbox da Lógica Fuzzy do Matlab, onde se aplicou a defuzzificação obtendo saídas numéricas que representam os níveis de vulnerabilidade em percentuais. 
Quadro 1 - Termos primários das variáveis presentes nas células ao longo das praias de Boa Viagem e Piedade .

\begin{tabular}{|c|c|c|c|c|c|c|}
\hline $\begin{array}{c}\text { Variáveis/ } \\
\text { Células }\end{array}$ & $\begin{array}{c}\text { Linha de } \\
\text { Costa }\end{array}$ & Pós-praia & $\begin{array}{c}\text { Urbani- } \\
\text { zação }\end{array}$ & Vegetação & $\begin{array}{c}\text { Enroca- } \\
\text { mento }\end{array}$ & $\begin{array}{c}\text { Benfeitoria } \\
\text { de Lazer }\end{array}$ \\
\hline 1 & Estável & Ampla & Moderada & Rasteira & Ausente & Ausente \\
\hline 2 & Estável & Ampla & Distante & Rasteira & Ausente & Ausente \\
\hline 3 & Moderada & Moderada & Distante & Rasteira & Ausente & Presente \\
\hline 4 & Moderada & Moderada & Distante & Arbustiva & Ausente & Presente \\
\hline 5 & Estável & Ampla & Moderada & Rasteira & Ausente & Adjacente \\
\hline 6 & Estável & Ampla & Moderada & Arbórea & Ausente & Ausente \\
\hline 7 & Estável & Ampla & Moderada & Arbustiva & Ausente & Ausente \\
\hline 8 & Moderada & Moderada & Moderada & Arbustiva & Ausente & Ausente \\
\hline 9 & Moderada & Moderada & Próxima & Arbórea & Moderado & Ausente \\
\hline 10 & Ausente & Ausente & Próxima & Ausente & Alto & Ausente \\
\hline 11 & Ausente & Ausente & Próxima & Ausente & Alto & Ausente \\
\hline 12 & Instável & Estreito & Próxima & Ausente & Alto & Ausente \\
\hline 13 & Instável & Estreito & Próxima & Arbustiva & Alto & Ausente \\
\hline 14 & Moderada & Moderada & Próxima & Rasteira & Moderado & Ausente \\
\hline 15 & Estável & Ampla & Moderada & Arbustiva & Ausente & Ausente \\
\hline 16 & Estável & Ampla & Moderada & Rasteira & Ausente & Ausente \\
\hline 17 & Estável & Ampla & Moderada & Arbustiva & Ausente & Ausente \\
\hline 18 & Estável & Ampla & Distante & Ausente & Ausente & Ausente \\
\hline 19 & Moderada & Moderada & Distante & Arbórea & Ausente & Ausente \\
\hline 21 & Moderada & Moderada & Moderada & Ausente & Ausente & Ausente \\
\hline
\end{tabular}

A Tabela 3 exibe entradas de cada célula que foi aplicada a lógica fuzzy constituída de 114 regras de inferência e seus resultados numéricos que indicam os níveis de vulnerabilidade, onde se analisou os níveis de vulnerabilidade à erosão. Em alguns casos, os níveis de vulnerabilidade podem apresentar duas situações, caracterizada pelo modelo fuzzy, porém pelo grau de pertinência verifica-se o nível dominante de vulnerabilidade à erosão no local analisado.

Como pode ser visto na Tabela 3 nas praias de Boa Viagem e Piedade existem locais onde a vulnerabilidade à erosão é classificada como: baixa, moderada, alta e muito alta. Vale salientar que a vulnerabilidade à erosão das praias caracterizada pela modelagem fuzzy tem o menor percentual $15 \%$ (baixo) e o maior em $63 \%$ (muito alto).

A Figura 4 apresenta a espacialização da vulnerabilidade das praias da Boa Viagem, Piedade e um trecho da praia do Pina, onde observa-se que no segmento ao Norte da construção do enrocamento da área de estudo aproximadamente 57,15\% apresenta vulnerabilidade baixa.

Bol. Ciênc. Geod., sec. Artigos, Curitiba, v. 19, nº 4, p.746-764, out-dez, 2013. 
Tabela 3 - Análise da entrada das variáveis presentes nas células ao longo das praias de Boa Viagem e Piedade.

\begin{tabular}{|c|c|c|c|c|}
\hline Células & Input & $\begin{array}{c}\text { Índice de } \\
\text { Vulnerabilidade }\end{array}$ & $\begin{array}{c}\text { Níveis de } \\
\text { Vulnerabilidade }\end{array}$ & $\begin{array}{c}\text { Níveis de } \\
\text { Vulnerabilidade } \\
\text { (Grau de } \\
\text { Pertinência) } \\
\end{array}$ \\
\hline 1 & {$\left[\begin{array}{llllll}80 & 80 & 60 & 0.5 & 0 & 80\end{array}\right]$} & $22,3 \%$ & Baixa/Moderada & Baixa \\
\hline 2 & {$\left[\begin{array}{lllllll}100 & 100 & 100 & 0.5 & 0 & 80\end{array}\right]$} & $15 \%$ & Baixa & Baixa \\
\hline 3 & {$\left[\begin{array}{lllllll}80 & 80 & 100 & 0.5 & 0 & 30\end{array}\right]$} & $22 \%$ & Baixa/Moderada & Baixa \\
\hline 4 & {$\left[\begin{array}{lllllll}60 & 60 & 100 & 1.5 & 0 & 30\end{array}\right]$} & $22,5 \%$ & Baixa/Moderada & Moderada \\
\hline 5 & {$\left[\begin{array}{llllll}60 & 60 & 70 & 0.5 & 0 & 40\end{array}\right]$} & $22 \%$ & Baixa/Moderada & Baixa \\
\hline 6 & {$\left[\begin{array}{llllll}40 & 40 & 60 & 5 & 0 & 50\end{array}\right]$} & $29,8 \%$ & Baixa/Moderada & Moderada \\
\hline 7 & {$\left[\begin{array}{llllll}40 & 40 & 50 & 2 & 0 & 50\end{array}\right]$} & $29,8 \%$ & Baixa/Moderada & Moderada \\
\hline 8 & {$\left[\begin{array}{llllll}40 & 40 & 50 & 1.5 & 0.5 & 60\end{array}\right]$} & $32,7 \%$ & Moderada/Alta & Moderada \\
\hline 9 & {$\left[\begin{array}{llllll}25 & 25 & 40 & 3 & 1 & 60\end{array}\right]$} & $37,6 \%$ & Moderada/Alta & Alta \\
\hline 10 & {$\left[\begin{array}{llllll}0 & 0 & 15 & 0 & 9 & 60\end{array}\right]$} & $54,6 \%$ & Alta/Muita Alta & Muito Alta \\
\hline 11 & {$\left[\begin{array}{llllll}0 & 0 & 15 & 0 & 10 & 70\end{array}\right]$} & $63 \%$ & Muito Alta & Muito Alta \\
\hline 12 & {$\left[\begin{array}{lllllll}15 & 15 & 15 & 0 & 10 & 70\end{array}\right]$} & $53,6 \%$ & Alta/Muito Alta & Muito Alta \\
\hline 13 & {$\left[\begin{array}{lllllll}15 & 15 & 15 & 3 & 10 & 75\end{array}\right]$} & $51,2 \%$ & Alta/Muito Alta & Alta \\
\hline 14 & {$\left[\begin{array}{llllll}40 & 40 & 15 & 0.5 & 1 & 75\end{array}\right]$} & $30,8 \%$ & Moderada/Alta & Moderada \\
\hline 15 & {$\left[\begin{array}{llllll}60 & 60 & 50 & 2 & 0.5 & 80\end{array}\right]$} & $22,5 \%$ & Baixa/Moderada & Moderada \\
\hline 16 & {$\left[\begin{array}{llllll}70 & 70 & 60 & 0.3 & 0 & 80\end{array}\right]$} & $22 \%$ & Baixa/Moderada & Baixa \\
\hline 17 & {$\left[\begin{array}{llllll}90 & 90 & 70 & 1 & 0 & 80\end{array}\right]$} & $21,2 \%$ & Baixa/Moderada & Baixa \\
\hline 18 & {$\left[\begin{array}{llllll}70 & 70 & 70 & 0 & 0 & 90\end{array}\right]$} & $20,6 \%$ & Baixa/Moderada & Baixa \\
\hline 19 & {$\left[\begin{array}{lllllll}50 & 50 & 80 & 1 & 0 & 90\end{array}\right]$} & $22,5 \%$ & Baixa/Moderada & Moderada \\
\hline 20 & {$\left[\begin{array}{lllllll}40 & 40 & 50 & 0 & 0 & 100\end{array}\right]$} & $32,8 \%$ & Moderada/Alta & Moderada \\
\hline 21 & {$\left[\begin{array}{lllllll}25 & 25 & 30 & 0 & 0 & 100\end{array}\right]$} & $46,5 \%$ & Alta/Muito Alta & Alta \\
\hline
\end{tabular}

$\mathrm{Na}$ análise e divisão dos setores ao longo das praias de Boa Viagem, Piedade e no trecho do Pina como mostrado anteriormente, observa-se que o setor 1 tem uma faixa de areia ampla e seguindo na direção sul essa faixa vai se estreitando. Observando a Figura 4 há um pequeno trecho em que a vulnerabilidade é moderada.

$\mathrm{O}$ setor 2 possui uma faixa de areia com $40 \mathrm{~m}$ que quando comparado com a Figura 4 a vulnerabilidade é modera em todo trecho deste setor, fato que condiz com a modelagem fuzzy. No setor 3 sua faixa de areia é adjacente a construção do enrocamento. Nesse setor a parte mais distante do enrocamento possui vulnerabilidade alta e a parte do setor mais próxima do enrocamento possui vulnerabilidade muito alta. $\mathrm{O}$ setor em que existe a construção do enrocamento 
apresenta uma vulnerabilidade muito alta, pois nesse setor a linha de costa é instável e a faixa de areia é estreita. O setor 4 quando na adjacência da construção do enrocamento, a vulnerabilidade é alta, na medida que se afasta passa a ter vulnerabilidade moderada e quando mais distante a vulnerabilidade é baixa. O setor 5 em que a faixa de areia já é bem mais estreita do que no setor 4 apresenta uma vulnerabilidade moderada e na medida que se aproxima da construção do enrocamento, passa a ter uma vulnerabilidade alta.

Figura 4 - Mapa de Vulnerabilidade Costeira das praias da Boa Viagem (Recife) e Piedade (Jaboatão dos Guararapes).

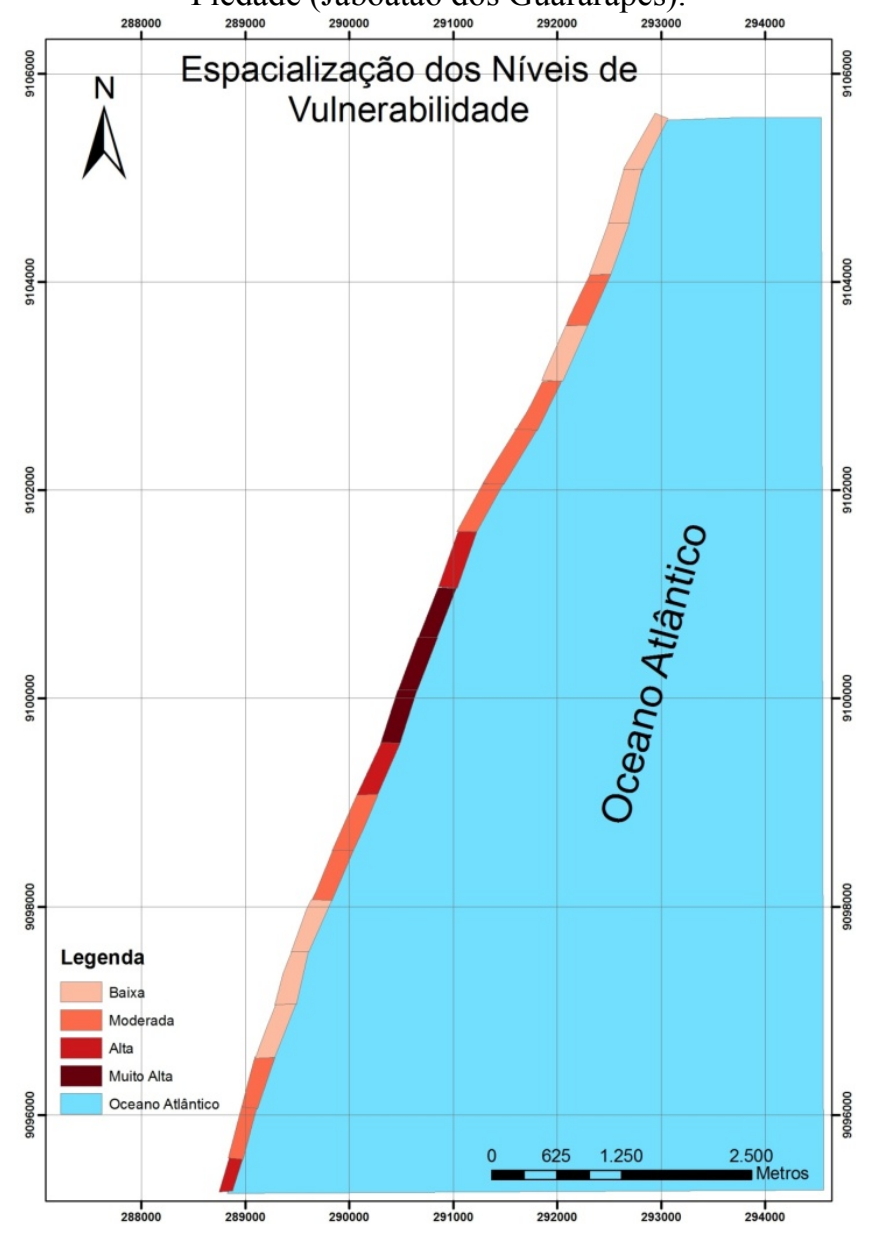

Bol. Ciênc. Geod., sec. Artigos, Curitiba, v. 19, nº 4, p.746-764, out-dez, 2013. 
Analisando os resultados obtidos nos setores observa-se que aproximadamente $33,3 \%$ da linha de costa apresenta vulnerabilidade baixa enquanto $14,26 \%$ da linha de costa apresenta vulnerabilidade muito alta. Para os termos intermediários: vulnerabilidade moderada e alta, a linha de costatem a extensão de $38,15 \%$ e $14,26 \%$, respectivamente como pode ser observado na Figura 4.

\section{CONCLUSÕES}

A vulnerabilidade à erosão costeira foi avaliada com uma metodologia semiquantitativa utilizada para identificar as mudanças ao longo do ambiente praial. Sua aplicação foi possível através da organização de um sistema de informações geográficas a partir de diferentes fontes cartográficas onde imagens de satélite artificiais de alta resolução para os anos de 2005 e 2011 serviram para identificar algumas das variáveis do modelo. O sistema GNSS foi utilizado para mapear a linha de costa e definir a variável chamada de processos costeiros de acordo com a análise temporal elaborada a partir dos dados obtidos para os anos 2007, 2009, 2010 e 2012.

Os dados de entrada que são quantificados identificam numericamente o comportamento da presença ou ausência de algumas variáveis. Neste trabalho foram utilizadas como variáveis: condições morfológicas, atributos naturais, processos costeiros e processos antrópicos conforme o critério associado a sua variável linguística fuzzy. Todavia, acredita-se que este modelo pode ser refinado, com a inserção de outras variáveis como: dados batimétricos, altimétricos, perfil de praia, dados oceanográficos entre outros que no caso seriam classificados como processos costeiros e poderiam contribuir com a acurácia do mapa de vulnerabilidade à erosão costeira.

Os segmentos das praias de Boa Viagem e Piedade apresentam características distintas em relação aos fatores determinantes da vulnerabilidade à erosão. Através da modelagem fuzzy das variáveis e das partições fuzzy foram identificados que $33,33 \%$ da linha de costa resultou em uma classificação da vulnerabilidade à erosão baixa, enquanto $14,26 \%$ da linha de costa apresentou uma vulnerabilidade alto e muito alta e a vulnerabilidade à erosão moderada apresentou uma extensão de $38,15 \%$ de linha de costa.

A partir destes resultados foi possível concluir e verificar que nas extensões menos vulneráveis foram detectadas uma pós-praia ampla, a linha de costa estável, a vegetação rasteira, a urbanização mais distante das áreas costeira e a ausência de processos antrópicos. Já as áreas mais vulneráveis à erosão relacionam-se com a influência antrópica na faixa de praia além de uma urbanização próxima da linha de costa, indicando que o modelo reflete a análise de um analista experiente, o que justificou a escolha da lógica fuzzy, indicando a funcionalidade do modelo proposto, com a vantagem da flexibilidade do modelo, podendo ser aplicado a outras áreas, independente da sua extensão.

Por fim pode-se concluir que ainda existem muitas questões que precisam ser analisadas e respondidas no que se refere ao diagnóstico e temas ligados a vulnerabilidade à erosão costeira. Através desta pesquisa percebeu-se que a lógica 
fuzzy possibilita uma alternativa para criar mapas de vulnerabilidade à erosão e se diferencia dos modelos analíticos clássicos que são definidos diretamente por uma fórmula e um limiar considerado inflexível quando comparado com a lógica fuzzy.

\section{REFERÊNCIAS BIBLIOGRÁFICAS}

ALMEIDA, H. R. R. C. Séries temporais de imagens suborbitais e orbitais de alta resolução espacial na avaliação da morfodinâmica praial no município do Cabo de Santo Agostinho -PE. Dissertação de conclusão de curso, (Mestrado em Ciências Geodésicas) UFPE, 2008.124p.

ANTUNES, A. F. B.; LINGNAU, C.; CENTENO, J. A. S. Object Oriented Analysis and Semantic Network for high Resolution Image Classification. Boletim de Ciências Geodésicas. Vol. 9, № 2, P. 233-242, 2003.

AURELIANO, J. T. Balnealidade das praias de Pernambuco: O núcleo Metropolitano. Dissertação de conclusão de curso (Mestrado em Gestão e Políticas Ambientais), UFPE, 2000. 113p.

BOAK, E. H.; TURNER, I. L. Shoreline Definition and Detection: A Review. Journal of Coastal Research. 21(4), P. 688-703, 2005.

BORUFF, B. J.; EMRICH, C.; CUTTER, S.L. Erosion hazard vulnerability of US coastal counties. Journal of Coastal Research, 21(5), P. 932-942, 2005.

BRYAN, B.; HARVEY, N.; BELPERIO, T.; BOURMAN, B. Distributed process modeling for regional assessment of coastal vulnerability to sea-level rise. Environmental Modeling and Assessment. 6, P.57-65, 2001.

CLARK, G. E.; MOSER, S. C.; RATICK, S. J.; DOW, K.; MEYER, W. B.; EMANI, S.; JIN, W.; KASPERSON, J. X.; KASPERSON, R. E.; SCHWARZ, H. E. Assessing the Vulnerability of Coastal Communities to Extreme Storms: The Case of Revere, MA., USA. Kluwer Academic Publishers: Printed in the Netherlands. 3, P. 59-82, 1998.

DANIEL, H. Replenishment versus retreat: the cost of maintaining Delaware's beaches. Ocean \& Coastal Management. 44, P. 87-104, 2001.

DOODY, J. P. Shoreline management - conservation, managementor restoration? National Coastal Consultants. P.407-419, 2001.

FERNANDES, R. T. Supervisão de um Sistema Híbrido eólico/diesel usando Lógica Fuzzy. Dissertação de conclusão de curso (Mestrado em Engenharia Elétrica), UFMS, 2005. 118p.

FERNANDES, E.; BASTOS, L. C.; MELLO, R. Apoio à decisão ao Planejamento do uso do espaço com a utilização de Sistemas de Informações Geográficas e Lógica Difusa. Revista Brasileira de Cartografia. № 62, P. 43-54, 2010.

GOMIDE, F. A. C.; GUDWIN, R. R. Modelagem, Controle, Sistemas e Lógica Fuzzy. SBA Controle \& Automação. Vol. 4, No 3, P. 97-115, 1994.

GONÇALVES, R. M.; AWANGE, J.; KRUEGER, C. P.; HECK, B.; COELHO, L. $\mathrm{S}$. A comparison between three short-term shoreline prediction models. Ocean \& Coastal Management, v. 69, p. 102-110, 2012. 
GONÇAlVES, R. M.; COELHO, L. D. S ; KRUEGER, C. P. ; HECK, B. Modelagem preditiva de linha de costa utilizando redes neurais artificiais. Boletim de Ciências Geodésicas , v. 16, p. 420-444, 2010.

HAPKE, C.; PLANT, N. Predicting coastal cliff erosion using a Bayesian probabilistic model. Journal of Marine Geology. 278, P. 140-149, 2010.

HARLEY, M. D.; TURNER, I.L.; SHORT, A.D.; RANASINGHE, R. Assessment and integration of conventional, RTK-GPS and image-derived beach survey methods for daily to decadal coastal monitoring. Journal of Coastal Engineering, 2011, №58, P. 194-205.

IBGE (Instituto Brasileiro de Geografia e Estatística). Mapa de distribuição da população 2010. Acessado em 13/07/2011. http://www.ibge.gov.br/home/ geociencias/geografia/mapas_doc1.shtm.

KERSTING, A. P. B.; CENTENO, J. A. S. Object Oriented Transmission Line Corridors Classification using LIDAR Technology and a Non-Metric Digital Camera. Boletim de Ciências Geodésicas, Vol. 13, № 2, P. 295-315, 2007.

LAZZAROTTO, D. R.; SLUTER, C. R.; DALMOLIN, Q. Avaliação da qualidade de base Cartográfica por meio de indicadores e Sistema de Inferência Fuzzy. Boletim de Ciências Geodésicas: Resumos. Vol. 11, № 2, P.297-299, 2005.

MALLMANN, D. L. B.; ARAÚJO, T. C. M. Vulnerabilidade do Litoral Sul de Pernambuco à erosão. Tropical Oceanography. V. 38, nº 2, P. 129-151, 2010.

MANSO, V. A. V.; COUTINHO, P. N.; GUERRA, N. C.; JUNIOR, C. F. A. S. Erosão e Progradação do Litoral Brasileiro: Pernambuco. Laboratório de Geologia e Geofísica Marinha - LGGM. Recife: Editorial da Universidade, 2006.

MARANDOLA JR, E; HOGAN, D. J. Vulnerabilidade do lugar vs. Vulnerabilidade sociodemográfica: implicações metodológicas de uma velha questão. Revista Brasileira de Estudos de População. Vol. 26, N 2, P. 161181, 2009.

MAZZER, A. M.; DILlENBURG, S. R.; SOUZA, C. R. G. Proposta de método para análise de vulnerabilidade à erosão costeira no sudeste da ilha de Santa Catarina, Brasil. Revista Brasileira de Geociências. 38(2), P.278-294, 2008.

MORAIS, J. O.; PINHEIRO, L. S.; CAVAlCANTE, A. A.; PAUlA, D. P.; SILVA, R. L. Erosão Costeira em Praias Adjacentes às Desembocaduras Fluviais: O Caso de Pontal de Maceió, Ceará, Brasil. Revista da Gestão Costeira Integrada. 8(2), P. 61-76, 2008.

RAPOSEIRO, P.D.; FERREIRA, J. C. R. A análise da vulnerabilidade e do risco de inundação como ferramenta de apoio à gestão dos territórios litorais sob pressão urbana. Pluris: Planejamento urbano regional integrado $e$ sustentável. P. 1-12, 2010.

REZENDE, S. O. Sistemas Inteligentes Fundamentos e Aplicações. $1^{\mathrm{a}}$ ed. Barueri SP: Manole, 2005.

ROCHA, C. P.; ARAÚJO, T. C. M.; MENDONÇA, F. J. B. Aplicação de técnicas de posicionamento GPS tridimensional para localizar linhas de costa: estudo 
de caso na praia de Boa Viagem, Recife-PE, Brasil. Revista da Gestão Costeira Integrada. 8(2), P. 127-137, 2008.

RYABCHUK, D.; SPIRIDONOV, M.; ZHAMOIDA, V.; NESTEROVA, E.; SERGEEV, A. Long term and short term coastal line changes of the Eastern Gulf of Finland: Problems of coastal erosion. J Coast Conserv. Springer Science. P. 1-10, 2010.

SEEBER, G. Satellite Geodesy: Foundations, Methods and Aplications. Berlin-New York, 2003, 589p. In: Walter de Gruyter. $2^{\text {nd }}$ revised edition.

SHAW, I.S.; SIMÕES, M.G. Controle e Modelagem Fuzzy. 2a ed. São Paulo: Blücher, 2007.

SILVA, M. A. Aplicação de Lógica Nebulosa para Previsão do Risco de Escorregamentos de Taludes em Solo Residual. Dissertação de conclusão de curso (Mestrado em Engenharia Civil), UERJ, 2008. 150 p.

SILVA, L. M. Modelagem Fuzzy como subsídios para a espacialização da vulnerabilidade costeira à erosão. Dissertação de conclusão de curso (Mestrado em Ciências Geodésicas), UFPE, 2013, 164 p.

SMITH, R. A. Beach resorts: A model of development evolution. Landscape and Urban Planning. N²1, P. 189-210, 1991.

TANAJURA, E. L. X.; KRUEGER, C. P.; GONÇALVES, R. M. Análise da acurácia dos métodos cinemáticos de posicionamento GPS em aplicações costeiras. Boletim de Ciências Geodésicas, v. 17, p. 23-36, 2011.

WU, S.; YARNAL, B.; FISHER, A. Vulnerability of coastal communities to sealevel rise: a case study of Cape May County, New Jersey, USA. Climate Research: Clim Res. Vol. 22, P. 255-270, 2002.

XIMENES, A. C.; ALMEIDA, C. M.; AMARAL, S.; ESCADA, M. I. S.; AGUIAR, A. P. D. Modelagem Dinâmica do desmatamento na Amazônia. Boletim de Ciências Geodésicas, Vol. 14, No 3, P. 370-391, 2008.

ZADEH, L. A. Fuzzy Sets, Inform. and Control. 8, P. 338-353, 1965.

(Recebido em março de 2013. Aceito em setembro de 2013). 